\title{
Adesão dos trabalhadores de enfermagem do hospital municipal Dr. Jair Braga aos equipamentos de proteção individual
}

Este estudo teve como objetivo avaliar a adesão dos trabalhadores de enfermagem aos equipamentos de proteção individual no Hospital Municipal Dr. Jair Braga localizado na cidade de Uauá/BA. Trata-se de um estudo descritivo, exploratório, transversal, de campo, com abordagem quantitativa. A população estudada fo composta por 20 profissionais de Enfermagem, divididos em: auxiliares de enfermagem e técnicos de enfermagem. Obtidos os seguintes resultados: $35 \%$ dos profissionais possuem faixa etária de 26 a 34 anos, $80 \%$ do sexo feminino, 100\% obtêm ensino médio completo e $35 \%$ são solteiros, $85 \%$ são técnicos de enfermagem, $30 \%$ possuem de 8 a 12 anos de tempo de serviço, $50 \%$ dos profissionais trabalham no setor de emergência, $60 \%$ dos profissionais possuem apenas 1 vínculo trabalhista, quando utilizar os EPIs, $70 \%$ dos profissionais referiram que não possuem, $100 \%$ referem que os EPIs são importantes para o trabalho de enfermagem, $90 \%$ nunca sofreram acidentes de trabalho por não utilizarem os EPIs, $60 \%$ referem quantidade suficiente de EPIs para os procedimentos, $40 \%$ não veem dificuldades para utilizarem os EPIs, $60 \%$ referem que os EPIs oferecidos são de boa qualidade, $100 \%$ não recebem capacitação, Os profissionais questionados referem não obterem capacitação e orientação quanto à adesão aos EPIs, o que torna uma vulnerabilidade para maiores riscos de infecções e acidentes no ambiente hospitalar, colocando em exposição a saúde tanto do profissional quanto a do cliente. Conclui-se que a adesão aos EPIs é um quesito fundamental no trabalho da equipe de enfermagem no combate à ocorrência dos acidentes de trabalhos e infecções hospitalares. É preciso que os profissionais se conscientizem dessa importante precaução e que as instituições proporcionem equipamento de qualidade, quantidade e adequados aos procedimentos a serem realizados.

\section{Accession of nursing workers at hospital municipal Dr. Jair Braga to personal protection equipment}

This study aimed to assess the adherence of nursing workers to personal protective equipment at the Municipal Hospital Dr. Jair Braga, located in the city of Uauá/BA. This is a descriptive, exploratory, cross-sectional, field study, with a quantitative approach. The studied population consisted of 20 nursing professionals, divided into: nursing assistants and nursing technicians. The following results are obtained: $35 \%$ of the professionals are aged between 26 and 34 years old, $80 \%$ female, $100 \%$ have completed high school and $35 \%$ are single, $85 \%$ are nursing technicians, $30 \%$ are 8 to 12 years old of service, $50 \%$ of professionals working in the emergency sector, $60 \%$ of professionals have only 1 job, when using PPE, $70 \%$ of referenced professionals who do not have it, $100 \%$ refer to PPE important for nursing work, $90 \%$ have never suffered work accidents for not using PPE, $60 \%$ refer to a sufficient amount of PPE for procedures, $40 \%$ do not see difficulties in using PPE, $60 \%$ refer that PPE offers good quality, $100 \%$ does not receive training, The professionals questioned refer to if they do not obtain training and guidance regarding adherence to PPE, or make them vulnerable to greater risks of infections and accidents in the hospital environment, put both health products on display professional and the client. Conclude that adherence to PPE is a fundamental problem in the work of the nursing team in combating the occurrence of work accidents and hospital infections. It is necessary for professionals to become aware of this important precaution and to provide equipment of quality, quantity and application to the procedures to be used.

Keywords: Protective equipment; Nursing; Work Accidents.

Reviewed anonymously in the process of blind peer.

Katyane Rodrigues Cardoso (id Centro Universitário UniAges, Brasil http://lattes.cnpq.br/7032693811180511 http://orcid.org/0000-0002-4223-3887 tiane.cardoso@hotmail.com

Ana Paula Gomes Soares Pereira (iD Universidade Estadual de Minas Gerais, Brasil http://lattes.cnpq.br/3631825469916595 http://orcid.org/0000-0002-1050-6035 anapaulagsoares@yahoo.com.br

Renan Sallazar Ferreira Pereira (ib) Universidade Federal do Tocantins, Brasil http://lattes.cnpq.br/8154326371029706 http://orcid.org/0000-0001-5140-4046 renansallazar@mail.uft.edu.br

\author{
Elvis das Neves de Souza \\ Centro Universitário UniAges, Brasil \\ http://lattes.cnpq.br/2100716023682132 \\ http://orcid.org/0000-0001-8652-4603 \\ elvis.nsouza@hotmail.com \\ Humberto Aparecido Faria (iD \\ Centro Universitário UniAges, Brasil \\ http://lattes.cnpq.br/3226370935768571 \\ http://orcid.org/0000-0002-9784-5506 \\ humbertofaria@yahoo.com.br \\ Tatiane Jesus dos Santos (iD \\ Centro Universitário UniAges, Brasil \\ http://lattes.cnpq.br/0910749444505327 \\ http://orcid.org/0000-0002-0603-5402 \\ tatianejsantos1993@hotmail.com
}

\author{
Christiano Miranda (iD) \\ Universidade Cruzeiro do Sul, Brasil \\ http://lattes.cnpq.br/4631433999709517 \\ http://orcid.org/0000-0003-2616-8744 \\ chmiranda1977@gmail.com \\ Daciane Souza dos Santos (iD \\ Centro Universitário UniAges, Brasil \\ http://lattes.cnpq.br/8792491734322092 \\ http://orcid.org/0000-0003-1010-5933 \\ dacianesouza@hotmail.com.br
}

Referencing this:

CARDOSO, K. R.; PEREIRA, A. P. G. S.; PEREIRA, R. S. F.; SOUZA, E N. FARIA, H. A.; SANTOS, T. J.; MIRANDA, C.; SANTOS, D. S.. Adesão dos trabalhadores de enfermagem do hospital municipal Dr. Jair Braga aos equipamentos de proteção individual. Scire Salutis, v.10, n.3, p.88-96, 2020. DOI: http://doi.org/10.6008/CBPC2236-9600.2020.003.0011

DOI: 10.6008/CBPC2236-9600.2020.003.0011 


\section{INTRODUÇÃO}

O Equipamento de Proteção Individual (EPI) caracteriza-se como todo dispositivo ou produto de uso individual utilizado pelo trabalhador que se destina à proteção contra riscos que ameaçam a sua segurança e saúde. O EPI colabora na realização dos trabalhos, minimizando os efeitos negativos acrescentados ao trabalhador através de riscos de acidentes ou transmissão de alguma doença (ANVISA, 2014).

De acordo com a Norma Regulamentadora - NR 6, considera-se Equipamento de Proteção Individual (EPI) todo dispositivo ou produto, de uso individual, utilizado pelo trabalhador, destinado à proteção de riscos suscetíveis de ameaçar a segurança e a saúde no trabalho. Este equipamento deve ser aprovado por órgão competente do Ministério do Trabalho e Emprego (MTE) e é de fornecimento gratuito e obrigatório aos empregados que dele necessitarem. Fabricante e importador, empregado e empregador têm obrigações com relação ao seu uso.

O fato dos profissionais aderirem ao uso de equipamentos de proteção está profundamente relacionado à percepção que eles têm acerca dos riscos a que estão expostos e da constante exposição a esses riscos. Ao aderirem ao uso dos EPIs, os profissionais estão adquirindo benefícios à sua saúde, proporcionando maior produtividade e promovendo a diminuição dos riscos de acidentes de trabalho. Mesmo tendo conhecimento sobre os riscos a que estão expostos, no ambiente hospitalar, nem sempre garante a adesão dos profissionais ao uso de medidas protetoras (SILVA et al., 2012).

Assim, de acordo com Campo et al. (2011) a adoção dos equipamentos de proteção pelos trabalhadores da saúde é considerada um desafio, uma vez que essa adoção é aceita teoricamente, mas ela ainda não permeia a prática diária com a mesma intensidade. Esse fato é resultante de uma série de fatores, tais como: a indisponibilidade/inadequação dos equipamentos de proteção individual (EPI), sobrecarga de trabalho, falta de capacitação quanto ao uso correto das medidas de biossegurança existentes a serem realizadas, bem como do próprio sentimento de invulnerabilidade e do hábito do uso.

Dessa forma, a grande preocupação com a falta do uso dos equipamentos de proteção individual é o risco ocupacional a que os trabalhadores de enfermagem estão expostos, relacionados ao desempenho de suas funções, uma vez que eles manipulam materiais biológicos e podem sofrer acidentes, pois o ambiente hospitalar é considerado insalubre, por agrupar os indivíduos portadores de diversas enfermidades infectocontagiosas, que se destacam como as principais fontes da transmissão de micro-organismos patogênicos, sendo encontradas as seguintes fontes de transmissão: fluidos corpóreos, sangue durante a realização dos procedimentos invasivos e através de manipulação de artigos e superfícies contaminadas (SOUZA, 2011).

Este estudo teve como objetivo avaliar a adesão dos trabalhadores de enfermagem na utilização dos equipamentos de proteção individual no ambiente hospitalar, recaindo sobre os impactos na saúde do trabalhador de enfermagem decorrente da não adesão da utilização dos equipamentos de proteção individual. 


\section{METODOLOGIA}

Trata-se de um estudo descritivo, exploratório, transversal, de campo, com abordagem quantitativa, realizada no Hospital Municipal de Uauá Dr. Jair Braga. A população pesquisada foi composta por 20 trabalhadores da enfermagem, sendo um total de 17 técnicos e 3 auxiliares, que atenderam ao critério de inclusão: estar presente na instituição no dia marcado para a coleta de dados profissionais em regime trabalhista estatutário.

A coletada de dados ocorreu em abril de 2015 após aprovação do Comitê de Ética da instituição sob parecer no 360215 respeitando ainda todas às exigências da Resolução 466/12, antiga Resolução 196/96, assim como autorização prévia da instituição hospitalar. Os participantes que estavam de comum acordo foram convidados para uma sala reservada para que de maneira individual fosse assinado o Termo de Consentimento Livre e Esclarecido em duas vias e respondessem aos questionários, condutas estas que tem como objetivo a garantia de privacidade do sujeito.

O instrumento de coleta de dados foi composto por variáveis sociodemográficas, profissionais e sobre a adesão aos equipamentos de proteção individual. Os dados coletados e explorados armazenados em banco de dados do Excel do pacote Microsoft Office 2010 e analisados por meio das medidas estatísticas descritivas simples, disposto em tabelas de agrupamento para cada variável extraída. Foram analisados e representados através de dados quantitativos para que se realize uma análise de conhecimento sobre o uso do equipamento de proteção individual entre os profissionais de enfermagem.

\section{RESULTADOS}

Em relação à faixa etária, mostra-se, na Tabela 1, que 3 profissionais (15\%) têm idade entre 18 e 26 anos, enquanto que 7 (35\%) apresentam idade entre 26 e 34 anos. Além disso, 5 (25\%) estão na faixa etária de 34 a 42 anos e 4 (20\%) têm entre 42 e 50 anos, apenas 1 (5\%) participante tem 50 anos ou mais.

Tabela 1: Distribuição dos profissionais de Enfermagem quanto aos dados sociodemográficos, Uauá/BA, 2015.

\begin{tabular}{lll}
\hline Variáveis & $\mathbf{n}$ & $\%$ \\
\hline Idade & & 15 \\
\hline 18 a 26 & 3 & 35 \\
26 a 34 & 7 & 25 \\
34 a 42 & 5 & 20 \\
42 a 50 & 4 & 5 \\
50 ou mais & 1 & 100 \\
\hline Total & 20 & \\
\hline Sexo & & $\mathbf{8 0}$ \\
\hline Feminino & 16 & $\mathbf{1 0 0}$ \\
Masculino & 4 & 100 \\
\hline Total & $\mathbf{2 0}$ & $\mathbf{1 0 0}$ \\
\hline Escolaridade & & \\
\hline Ensino Médio completo & 20 & 35 \\
\hline Total & $\mathbf{2 0}$ & 30 \\
\hline Estado civil & & 10 \\
\hline Solteiro(a) & 7 & 10 \\
Casado(a) & 6 & 15 \\
Separado(a) & 2 & $\mathbf{1 0 0}$ \\
\hline Viúvo(a) & 2 & \\
\hline Toutros & 3 & $\mathbf{2 0}$ \\
\hline
\end{tabular}


Em relação à faixa etária, mostra-se, na Tabela 1, que 3 profissionais (15\%) têm idade entre 18 e 26 anos, enquanto que 7 (35\%) apresentam idade entre 26 e 34 anos. Além disso, 5 (25\%) estão na faixa etária de 34 a 42 anos e 4 (20\%) têm entre 42 e 50 anos, apenas 1 (5\%) participante tem 50 anos ou mais. Com relação ao gênero, a partir dos números obtidos, observa-se que o sexo feminino tem maior predominância, sendo16 (80\%), enquanto que o sexo masculino corresponde a 4 (20\%).

Quando questionados sobre a escolaridade, verifica-se que todos profissionais possuem o ensino médio completo 20 (100\%), tanto os técnicos como os auxiliares de enfermagem. Quanto ao estado civil, identifica-se que entre os profissionais técnico e auxiliares de enfermagem encontrados, 8 (30\%) são casados, 5 (35\%) são solteiros, viúvos $2(10 \%)$, separados 2 (10\%) e outros $3(15 \%)$.

Tabela 2: Distribuição dos profissionais de Enfermagem quanto ao perfil profissional, Uauá/BA, 2015.

\begin{tabular}{|c|c|c|}
\hline Variáveis & $\mathbf{n}$ & $\%$ \\
\hline \multicolumn{3}{|l|}{ Profissão } \\
\hline Técnico de Enfermagem & 17 & 85 \\
\hline Auxiliar de Enfermagem & 3 & 15 \\
\hline Total & 20 & 100 \\
\hline \multicolumn{3}{|l|}{ Tempo de serviço (anos) } \\
\hline 1 a 4 & 1 & 5 \\
\hline 4 a 8 & 2 & 10 \\
\hline 8 a 12 & 6 & 30 \\
\hline 12 a 16 & 5 & 25 \\
\hline 16 a 20 & 4 & 20 \\
\hline 20 ou mais & 2 & 10 \\
\hline Total & 20 & 100 \\
\hline \multicolumn{3}{|l|}{ Setor de trabalho } \\
\hline C. Médica & 6 & 30 \\
\hline Maternidade & 2 & 10 \\
\hline C. Pediátrica & 2 & 10 \\
\hline Emergência & 10 & 50 \\
\hline Total & 20 & 100 \\
\hline \multicolumn{3}{|l|}{ Vínculos } \\
\hline 1 & 12 & 60 \\
\hline 2 ou mais & 8 & 40 \\
\hline Total & 20 & 100 \\
\hline
\end{tabular}

No que se refere à categoria profissional, a percentagem de profissionais que responderam ao questionário, pertencentes à categoria de técnico de enfermagem, é a de maior representatividade, com 17 (85\%), seguida da dos auxiliares de enfermagem, 3(15\%). Quanto ao tempo de atuação, constatou-se que 1(5\%) profissional possui 4 anos, 2 (10\%) profissionais 4 a 8 anos, 6 (30\%)profissionais 8 a 12 anos, 5 (25\%) profissionais de 12 a 16 anos, 4 (20\%) profissionais de 16 a 20 anos, 2 (10\%) profissionais 20 ou mais anos de atuação na área.

Ainda seguindo o perfil profissional, são descritos na Tabela 2 dados sobre a distribuição dos profissionais por setores de trabalho. Na clínica médica são lotados 6 (30\%) dos profissionais, na maternidade 2 (10\%), clínica pediátrica 2 (10\%), emergência 10 (50\%). Acerca do perfil dos profissionais: 12(60\%) dos profissionais questionados têm apenas 1 vínculo e 8 (40\%) têm 2 ou mais.

A Tabela 3 dispõe sobre o turno de trabalho dos profissionais de Enfermagem entrevistados, observase que 2 (10\%) executam suas atividades das 7 às 13h, também $2(10 \%)$ dos profissionais trabalham das 13 às $19 \mathrm{~h}$, diariamente, 8 (40\%) desempenham suas funções das 19 às $7 \mathrm{~h}$ em plantões alternados, e 8 (40\%) 
também realizam seu trabalho em plantões alternados das 19 às $7 \mathrm{~h}$.

Tabela 3: Distribuição dos dados referentes ao horário de trabalho, orientações, importância dos EPIs e acidente de trabalhos pela equipe de Enfermagem, Uauá/BA, 2015.

\begin{tabular}{lll}
\hline Variáveis & $\mathbf{n}$ & $\%$ \\
\hline Horário de Trabalho & 2 & 10 \\
\hline 7h às 13h & 2 & 10 \\
13h às 19h & 8 & 40 \\
19h às 7h (par) & 8 & 40 \\
19h às 7h (ímpar) & $\mathbf{2 0}$ & $\mathbf{1 0 0}$ \\
\hline Total & & 70 \\
\hline Orientação ao uso de EPI & 14 & 30 \\
\hline Sim & 6 & $\mathbf{1 0 0}$ \\
Não & $\mathbf{2 0}$ & \\
\hline Total & & 90 \\
\hline Importância do EPI & 18 & 10 \\
\hline Sim & 2 & $\mathbf{1 0 0}$ \\
Não & $\mathbf{2 0}$ & \\
\hline Total & & 90 \\
\hline Acidentes de trabalho & 18 & 10 \\
\hline Sim & 2 & $\mathbf{1 0 0}$ \\
\hline Não & $\mathbf{2 0}$ & \\
\hline
\end{tabular}

Dos referentes à orientação de como e quando utilizar os EPIs. Dos profissionais questionados, 14 (70\%) refere ter tido orientações de como e quando utilizar os EPIs nos procedimentos, já 6 (30\%) referiram não ter tido orientações quanto à problemática. Quanto à importância do uso dos EPIs, os dados mostram que 20 (100\%) dos profissionais confirmaram que os EPI são de fundamental importância para o trabalho dos técnicos e auxiliares de enfermagem. No que se refere aos dados sobrea os acidentes de trabalho por não utilizar os EPIs, 18 (90\%) dos profissionais afirmam que nunca sofreram acidentes de trabalho, enquanto que $2(10 \%)$ dos profissionais informam que já sofreram acidentes no trabalho decorrentes do não uso de EPI.

Os dados evidenciaram que $17(77,4 \%)$ dos profissionais nunca sofreram acidentes de trabalho, contudo, $2(9,1 \%)$ afirmaram já terem sofrido algum tipo de acidente de trabalho, e não receberam nenhum tipo de assistência. Apenas 1 (4,5\%) sofreu acidente de trabalho e foi bem atendido. Outros 2 participantes relatam ter sofrido AT, sendo que $1(4,5 \%)$ não notificou e apenas $1(4,5 \%)$ não esclareceu se houve assistência e/ou qual foi a assistência recebida.

Tabela 4: Distribuição dos dados referentes à quantidade de EPI, se são suficientes para todos os procedimentos, bem como existência de capacitação na instituição para os profissionais, Uauá/BA, 2015.

\begin{tabular}{lll}
\hline Variáveis & $\mathbf{n}$ & $\%$ \\
\hline Quantidade de EPI suficiente para todos os procedimentos & & 60 \\
\hline Sim & 12 & 40 \\
Não & 8 & 100 \\
\hline Total & 20 & \\
\hline Existência de capacitação na instituição para os profissionais & & 100 \\
\hline Não & 20 & 100 \\
\hline Total & $\mathbf{2 0}$ & \\
\hline
\end{tabular}

Na Tabela 4, é visto a distribuição dos dados referentes à quantidade de EPI, se são suficientes para todos os procedimentos, $12(60 \%)$ responderam que sim e $8(40 \%)$ que não consideram a quantidade ofertada pela instituição suficiente. 
Tabela 5: Distribuição dos dados referentes à principal dificuldade para o uso dos EPI, Uauá/BA, 2015.

\begin{tabular}{lcl}
\hline Variáveis & $\mathbf{n}$ & $\%$ \\
\hline \multicolumn{2}{l}{ Principal dificuldade para o uso de EPI } & \\
\hline Incômodo e interferência & 3 & 15 \\
Inexistência e qualidade & 2 & 10 \\
Falta de tempo e situações de & & \\
emergências & 1 & 5 \\
Falta de incentivo ao uso & 2 & 10 \\
Nenhum & 12 & 60 \\
\hline Total & $\mathbf{2 0}$ & $\mathbf{1 0 0}$ \\
\hline
\end{tabular}

$\mathrm{Na}$ Tabela 5, são observadas as respostas obtidas pelos profissionais quando questionados sobre as principais dificuldades na utilização dos EPIs. Assim sendo, nota-se que 2 (10\%) dos entrevistados referemse às barreiras relacionadas à inexistência e qualidade dos EPIs como maior dificuldade para seu uso, 12 (60\%) não veem empecilhos quanto ao uso dos EPIs, 3 (15\%) dos profissionais identificam o incômodo e a interferência durante o uso como principal problema, 1 (5\%) destaca a falta de tempo e situações de emergência como agravantes à utilização dos equipamentos e $2(10 \%)$ dos participantes citam a falta de incentivo ao uso como desencadeador da baixa adesão aos materiais.

\section{DISCUSSÃO}

Os dados demonstraram que $35 \%$ dos profissionais tem entre 26 e 34 anos de idade. Em um estudo acerca do quesito idade, percebe-se que grande parte dos profissionais pesquisados (47\%)tem entre 20 e 30 anos de idade e enquadram-se na categoria de adultos jovens. A força de trabalho dos profissionais de Enfermagem, no Brasil, majoritariamente, concentra-se na faixa etária de 26 a 50 anos, sendo que a grande maioria está na faixa de 26 a 35 anos, a qual representa $35,98 \%$ do total dos profissionais de enfermagem do Brasil (MACHADO et al., 2015).

Quanto aos profissionais com idade mais elevada, embora esse fato propicie uma série de benefícios para o processo de produção em saúde, alguns autores advertem que a alta segurança na realização de procedimentos pode tornar-se perigosa, pois o excesso de confiança na rotina de trabalho leva à banalização dos riscos existentes. Isso pode contribuir para o aumento da exposição dos profissionais e dos pacientes a riscos com a não adesão adequada às precauções, ou seja, aos Equipamentos de Proteção Individual (MACHADO et al., 2015).

A predominância feminina na enfermagem é compartilhada por outros autores, reproduzindo a característica histórica da enfermagem, profissão exercida quase que exclusivamente por mulheres desde os seus primórdios. Há relato na literatura que a predominância dos trabalhadores nos hospitais é do sexo feminino, principalmente, na enfermagem, explicada em função do arquétipo, atribuído às mulheres.

Analisando a equipe de enfermagem, notou-se que os técnicos e auxiliares são os profissionais com maior número no ambiente hospitalar, que, durante a jornada de trabalho, estão mais expostos a riscos devido à caracterização de suas atividades. Os dados demonstram que a maior parte dos profissionais apresenta de 8 a 20 anos de experiência na área. Pesquisadores, como Faria et al. (2019) sugerem a hipótese de que trabalhadores com mais tempo de serviço e experiência possam se sentir mais seguros e, de certa 
forma, negligenciar certas precauções, por confiar demasiadamente em sua destreza, acidentando-se, algumas vezes, por não aderirem ao uso dos EPIs.

A ocorrência de erros na utilização de EPI está ligada, dentre outros fatores, ao tempo de serviço, visto que, constantemente, os profissionais ingressam no mercado de trabalho sem conhecimento dos cuidados necessários para evitar a exposição aos riscos da rotina do serviço, permanecendo sem treinamento, após a admissão, sobre os fatores de risco presentes, ou, por já trabalharem por muitos anos, acabam adquirindo vícios profissionais, ou achando que nada de errado acontecerá (SOUZA et al., 2010).

Observa-se que o setor com maior número de profissionais é a emergência considerando ser um setor com maior fluxo de atendimento. A emergência é um setor de alta complexidade, em que se exige maior atenção com relação à dinâmica de trabalho por parte dos profissionais nele inseridos. Despertar nos trabalhadores a consciência da importância do uso correto dos EPIs é pertinente, uma vez que, assim, eles poderão atender às exigências de trabalho em um setor emergencial, além de que o seu ambiente de trabalho não será fonte de riscos e de doenças ocupacionais e sim fonte de prazer e realização profissional (BATISTONI et al., 2011).

A categoria de maior representatividade profissional foi a da enfermagem, que evidenciou que, dos pesquisados, 70,4\% possuem apenas um emprego, sendo eles, auxiliares de enfermagem e técnicos de enfermagem. $O$ fato de o trabalhador possuir mais de um vínculo empregatício pode ser um fator contribuinte para a ocorrência do acidente de trabalho, devido à sobrecarga de trabalho. Quando o profissional obtém apenas um vínculo, torna possível um trabalho com maior qualidade e com boa produtividade.

De modo geral, os trabalhadores de enfermagem se encontram expostos a uma diversidade e simultaneidade de cargas de trabalho. Quanto à equipe de enfermagem, algumas dificuldades a impedem de cuidar-se. Tais dificuldades se relacionam com o tipo de trabalho e a carga horária a ser cumprida, atribuições cansativas, turnos rotativos, manipulação de substâncias tóxicas e presença de fatores de risco de várias naturezas. A nosso ver, o regime de turnos e plantões abre espaços para duplos empregos e jornadas de trabalho, comum entre os profissionais de saúde, especialmente, num país em que os baixos salários predominam. Esta prática potencializa, decerto, a ação de fatores que por si só danificam a integridade física e psíquica do profissional de enfermagem (BATISTONI et al., 2011).

A orientação e supervisão do trabalho da enfermagem são fundamentais para os cuidados prestados, pois, a falta de orientação pode comprometer o trabalho da enfermagem, proporcionando risco à saúde do profissional e do cliente. A padronização e a socialização das rotinas, quanto ao uso e manuseio dos equipamentos de proteção, são imprescindíveis para que os profissionais tenham subsídios necessários para promover a segurança no ambiente de trabalho, orientar as práticas de adoções de comportamentos adequados no uso dos EPIs são de fundamental importância em relação também ao controle e à prevenção de acidentes ocupacionais nos serviços de saúde (SOUZA et al., 2011).

Nessa perspectiva, estudos demonstram que os profissionais têm conhecimento de biossegurança, pois afirmam fazer uso dos EPIs e reconhecem a importância da utilização dos mesmos no ambiente de 
trabalho, tratando em suas justificativas de questões de proteção e segurança no decorrer das atividades laborais (SOUSA, 2012).

Diversos autores afirmam que a maior frequência de acidentes de trabalho em hospitais sucede na enfermagem e defendem este argumento porque os trabalhadores estão expostos a riscos advindos do desenvolvimento de atividades assistenciais diretas e indiretas, cuidados prestados diretamente a pacientes e em organização. Possivelmente, a maior frequência de acidentes entre os trabalhadores de enfermagem, quando comparadas a outras categorias profissionais, decorre da complexidade do processo de trabalho da enfermagem. Dentre os trabalhadores da saúde, a equipe de enfermagem é quem convive mais tempo com os pacientes, realizando cuidado direto aos doentes nas 24 horas-dia, de todo o ano (NEVES et al., 2011).

O papel das instituições é prover as unidades e os setores de materiais e equipamentos de qualidade, na quantidade apropriada. O serviço de saúde deve garantir mecanismos de prevenção dos riscos de acidentes de trabalho, incluindo o fornecimento de Equipamentos de Proteção Individual - EPI, em número suficiente e compatível com as atividades desenvolvidas pelos trabalhadores (ANVISA, 2011).

A capacitação do pessoal de enfermagem sobre o uso dos EPIs na prevenção de acidentes, no combate às infecções hospitalares e cruzadas, limita-se à transmissão de informações e não à conscientização intensiva. Visto isso, é necessária a criação de espaços para que trabalhadores de enfermagem discutam questões relativas às condições de trabalho e que minimizem efetivamente contra os riscos. Nesta perspectiva de compreensão, os profissionais devem, a partir de suas experiências, reconhecerem-se no processo de trabalho e receberem e cambiarem conhecimentos sobre prevenção de acidentes e manutenção da saúde no trabalho, com apoio e presença de serviços de educação continuada (LIMA, 2013).

A adesão ao uso dos EPIs está diretamente relacionada à percepção que os profissionais têm acerca dos riscos aos quais estão expostos e da susceptibilidade a esses riscos. Desse modo, medidas de cunho educativo podem ser uma alternativa para melhoria do serviço prestado por esses sujeitos, no que se refere à adesão às precauções-padrão. Muitas vezes, a formação dispensada ao profissional técnico e auxiliar de enfermagem não contempla conteúdos relativos à problemática dos riscos a que estão expostos, com ênfase nas medidas de prevenção e controle (BATISTONI et al., 2011).

Os Acidentes de Trabalho (AT) estão diretamente ligados a vários fatores, dentre eles, a não utilização de EPI. Acredita-se que a baixa adesão ao uso destes pode estar vinculada à falta de orientação da equipe de enfermagem sobre a importância da sua utilização; ao não-fornecimento dos equipamentos pela empresa contratante; pelos profissionais não gostarem e sentirem-se incomodados com a utilização dos mesmos; como também por esquecimento e falta de hábito (CARVALHO et al., 2018).

\section{CONCLUSÕES}

A identificação dos problemas é imprescindível para a elaboração de estratégias de ação que visem à melhoria da qualidade de serviço prestado pela instituição e pela equipe de enfermagem. A elaboração de estratégias de intervenção deverá ser capaz de aprimorar a conduta dos profissionais de enfermagem, ou seja, de aumentar a adesão destes profissionais aos EPIs, para isso, requer quesitos como a capacitação, 
orientação e supervisão a ser executada pelo enfermeiro na instituição hospitalar, direcionado aos trabalhadores de enfermagem com a finalidade de discutir o assunto e esclarecer dúvidas, bem como capacitação de atualização. Tais medidas visam proporcionar uma percepção maior pelos profissionais na adesão ao uso de EPI e, consequentemente, a proteção e segurança destes trabalhadores.

Conclui-se que a adesão aos EPIs é um quesito fundamental no trabalho da equipe de enfermagem no combate à ocorrência dos acidentes de trabalhos e infecções hospitalares. É preciso que os profissionais se conscientizem dessa importante precaução e que as instituições proporcionem equipamento de qualidade, quantidade e adequados aos procedimentos a serem realizados, além de capacitação aos profissionais quanto à adesão aos Equipamentos de Proteção Individual.

\section{REFERÊNCIAS}

ANVISA. Agência Nacional de Vigilância Sanitária. NRR 32: Equipamento de Proteção Individual. ANVISA, 2011.

ANVISA. Agência Nacional de Vigilância Sanitária. Segurança no ambiente hospitalar. ANVISA, 2014.

BATISTONI, E. A.; BARBOSA, D.; SANTOS, L. H. G.; ANDREAZZI, D.. Importância do EPI: percepção da equipe de enfermagem na sala de emergência. Revista Eletrônica Acervo Saúde, Ouro Fino, v.2, p.55-69, 2011.

BRASIL. Secretaria de Trabalho. NR 6: equipamento de proteção individual/EPI. Brasília: Secretaria de Trabalho, 2001.

CAMPO, S. F.; VILAR, M. A.; VILAR, D. A.. Biossegurança: Conhecimento e Adesão às Medidas de Precauções Padrão num Hospital. Rev. Bras. Ciênc. Saúde, v.15, n.4, p.415-420, 2011. DOI: http://doi.org/10.4034/RBCS.2011.15.04.06

CARVALHO, D. C.; ROCHA, J. C.; GIMENES, M. C. A.; SANTOS, E. C.; VALIM, M. D.. Acidentes de trabalho com material biológico na equipe de enfermagem de um hospital do Centro-Oeste brasileiro. Esc. Anna Nery, Rio de Janeiro, v.22, n.1, p.e20170140, 2018

FARIA, L. B. G.; SANTOS, C. T. B.; FAUSTINO, A. M.; OLIVEIRA, L. M. A. C.; CRUZ, K. C. T.. Conhecimento e adesão do enfermeiro às precauções padrão em unidades críticas. Texto Contexto: Enferm., Florianópolis, v.28, 2019. DOI: https://doi.org/10.1590/1980-265x-tce-2018-0144

LIMA, J. P. B.. A utilização de equipamentos de proteção individual pelos profissionais de Enfermagem: práticas relacionadas com o uso de luvas. 2013.
MACHADO, M. H.; AGUIAR FILHO, W.; LACERDA, W. F.; OLIVEIRA, E.; LEMOS, W.; WERMELINGER, M.; VIEIRA, M.; SANTOS, M. R.; SOUZA JÚNIOR, P. B.; JUSTINO, E. BARBOSA, C.. Características Gerais Da Enfermagem: O Perfil Sócio Demográfico. Enferm. Foco, v.7, p.9-14, 2015.

NEVES, H. C. C.; SOUZA, A. C. S.; MEDEIROS, M.; MUNARI, D. B.; RIBEIRO L. C. M.; TIPPLE, A. F. V.. Segurança dos trabalhadores de enfermagem e fatores determinantes para adesão aos equipamentos de proteção individual. Rev. Latino-Am. Enfermagem, v.19, n.2, p.354-361, 2011. DOI: https://doi.org/10.1590/S0104-11692011000200018

SILVA, G. S.; ALMEIDA, A. J.; PAULA, V. S.; VILLAR, L. M.. Conhecimento e utilização de medidas de precaução-padrão por profissionais de saúde. Esc. Anna Nery, v.16, n.1, 2012. DOI: http://dx.doi.org/10.1590/S1414-81452012000100014

SOUZA, M. C. M. R.; FREITAS, M. I. F.. Representações de profissionais da atenção primária sobre risco ocupacional de infecção pelo HIV. Rev. Latino-Am. Enfermagem, v.18, n.4, p.748-754, 2010. DOI: https://doi.org/10.1590/S010411692010000400013

SOUZA, E. L. V.; NASCIMENTO, J. C.; CAETANO, J. A.. Uso dos equipamentos de proteção individual em unidade de terapia intensiva. Revista de Enfermagem Referência, v.3, n.4, p.125-133, 2011.

SOUZA, R. J.. A percepção dos profissionais de enfermagem que pode gerar doenças/agravos a seu estado de saúde. 2011.

SOUSA, R. C.. Uso de equipamentos de proteção individual pelos profissionais de enfermagem. Picos, 2012.

A CBPC - Companhia Brasileira de Produção Científica (CNPJ: 11.221.422/0001-03) detém os direitos materiais desta publicação. Os direitos referem-se à publicação do trabalho em qualquer parte do mundo, incluindo os direitos às renovações, expansões e disseminações da contribuição, bem como outros direitos subsidiários. Todos os trabalhos publicados eletronicamente poderão posteriormente ser publicados em coletâneas impressas sob coordenação da Sustenere Publishing, da Companhia Brasileira de Produção Científica e seus parceiros autorizados. Os (as) autores (as) preservam os direitos autorais, mas não têm permissão para a publicação da contribuição em outro meio, impresso ou digital, em português ou em tradução. 